\title{
The Simultaneous Processing of Multiple Specimens from the Same Patient to Improve the Efficiency of Mohs Surgery
}

\author{
Tonja Godsey ${ }^{1}$, Rebecca Jacobson ${ }^{1}$, Hugh Gloster* \\ Department of Dermatology, University of Cincinnati, Ohio
}

Received: 08 August, 2016; Accepted: 17 August, 2016; Published: 27 August, 2016

*Corresponding author: Hugh M. Gloster, Jr, MD, 3590 Lucille Dr, suite 1600, Cincinnati, Ohio 45213, Telephone: 513-475-7630, Fax: 513-487-1000; Email: hgloster@yahoo.com

\begin{abstract}
Background: Mohs Micrographic Surgery (MMS) may be a lengthy procedure, particularly when it is necessary to process multiple specimens in the same patient. This scenario commonly occurs when a patient undergoes MMS for two or more tumors on the same operative day or when it is necessary to re-excise multiple different specimens from the periphery and/or center of a defect.

Purpose: The purpose of this manuscript is to describe a method of simultaneous processing multiple small tissue specimens of similar consistency from the same patient, which allows the Mohs surgeon to view two or more specimens at once on one slide.

Conclusions: This technique reduces the number of glass slides utilized and increase the rapidity with which frozen sections can be prepared and examined, thus adding to the growing body of literature describing more efficient measures of frozen section processing in Mohs Micrographic Surgery.
\end{abstract}

\section{Short Communication}

Mohs micrographic surgery (MMS) may be a lengthy procedure, particularly when it is necessary to process multiple specimens in the same patient. This scenario occurs when a patient undergoes MMS for two or more tumors on the same operative day or when it is necessary to re-excise multiple different specimens from the periphery and/or center of a defect after microscopic examination of an excisional specimen reveals residual, multifocal tumor. In this article, we will discuss the simultaneous processing multiple specimens from the same patient, which allows the Mohs surgeon to view two or more specimens at once on one slide, thus reducing the number of glass slides utilized and increasing the rapidity with which frozen sections can be prepared and examined.

It is not uncommon for a patient to present to the Mohs surgeon for the treatment of more than one tumor on the same operative day. Embedding, sectioning, and mounting each specimen separately on different glass slides can be time-consuming for the histotechnician and wasteful of slides, especially if each excisional specimen is divided into two or more pieces prior to frozen section processing. Alternatively, small (usually 2 to 4 specimens depending on their size) excisional specimens from anatomic sites of similar consistency on the same patient may be sectioned together and mounted on one glass slide by the single section method. The single section method saves time by omitting the division each specimen into smaller sections, which reduces the number of specimens that must be cut, stained, and cover slipped by the technician and interpreted by the surgeon [1-3]. This method permits the surgeon to view all of the specimens at once and increases the rapidity of frozen section preparation and examination (Figure 1).

The same concept described above can be applied to instances when, after microscopically examining and mapping an excised layer of tissue, it necessary to re-excise multiple, small pieces of tissue at the periphery of the defect due to residual epidermal tumor (Figure 2). This scenario commonly occurs during the treatment of certain neoplasms that exhibit subclinical horizontal spread through the epidermis, such as squamous cell carcinoma in situ, malignant melanoma in situ, or multifocal superficial basal cell carcinoma. Multiple small tissue specimens (typically two to four in number) may be embedded together in frozen

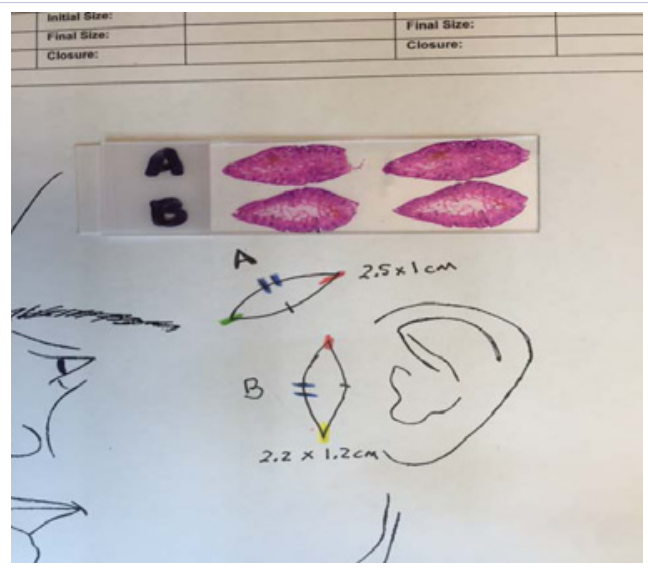

Figure 1: Specimens from two different sites labeled A and B are cut as a single sections and mounted on one slide. The nicks and colored dyes on the specimens correspond precisely to the Mohs map. The sizes of the specimens are indicated on the Mohs map. 
section medium on the same cryostat chuck (Figure 3), sectioned together, and mounted on the same glass slide (Figure 4) [4]. This method allows the Mohs surgeon to rapidly view all of the specimens at once on one slide.

The simultaneous processing of multiple specimens is ideally suited for small and medium sized specimens of similar consistency, which usually occurs when specimens are excised from the same anatomic area. In other words, the specimens should have similar dermal thickness and adipose content Modest sized specimens of similar consistency will freeze at a similar rate and yield higher quality frozen sections without holes or tears. The number of specimens that may be sectioned and mounted on a single glass slide depends on the number and size of each of the specimens, since they all must fit on one $2.5 \mathrm{x}$ $7.5 \mathrm{~cm}$ glass slide together. The diameter of the cryostat chuck is not a size-limiting factor, because specimens that are larger than the chuck may be successfully processed and sectioned if the specimen head can travel full circle across the blade and back up to its original position.

There are potential risks that are involved with the simultaneous processing of multiple specimens, particularly for an inexperienced histotechnician. First, there is the potential of deeper facing of the tissue block in order to achieve complete

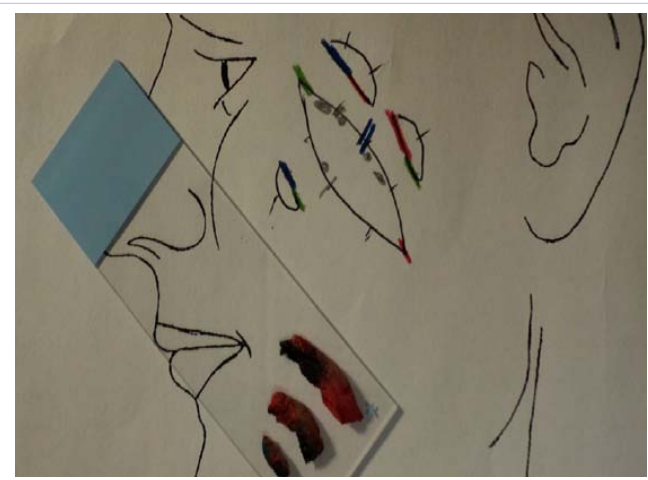

Figure 2: Multiple small pieces of tissue were excised from the periphery of this defect during the second stage of Mohs surgery.

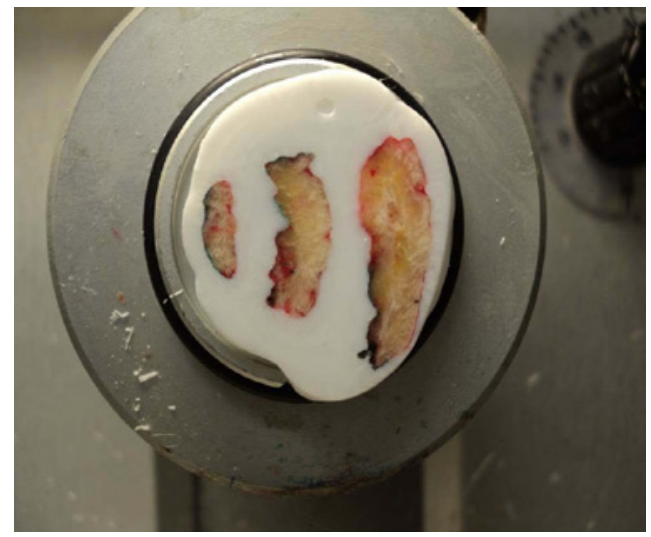

Figure 3: Three small tissue specimens are embedded together in frozen section medium on the same chuck.

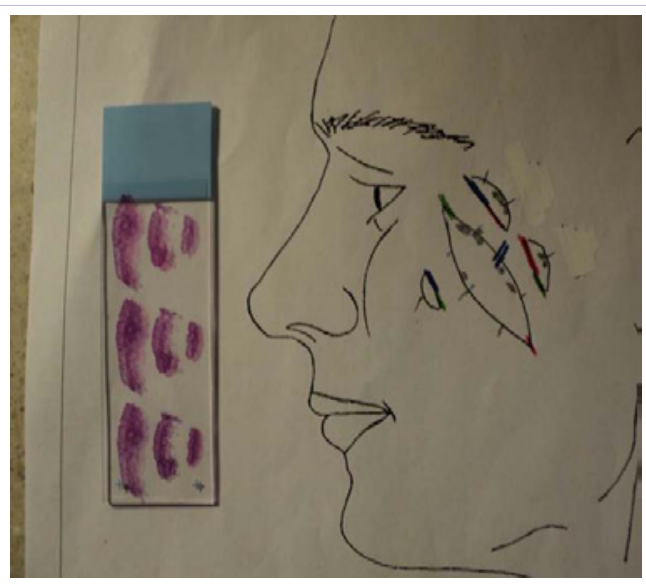

Figure 4: Three small specimens have been mounted on the same glass slide for examination during the second stage of Mohs surgery. Each specimen was harvested from the periphery of the defect as indicated by the Mohs map.

epidermis of all specimens, which can increase the probability of a false positive microscopic analysis of the excisional specimen and of smaller pieces being consumed or chunked out. The risk of excessive facing can be minimized by utilizing a cryostat equipped with an x-y axis chuck head. Second, it may be more difficult to align multiple specimens appropriately on the chuck so that they are exactly flush. Utilizing an alignment apparatus and a glass slide to mount specimens in the frozen section medium can reduce the potential for improper alignment of specimens on the chuck. Finally, there is more potential for error with inking and mapping of multiple specimens. Consequently, meticulous mapping, labeling and documentation are needed to reduce the risk of laboratory error.

In conclusion, the simultaneous processing multiple specimens from the same patient allows the Mohs surgeon to view two or more specimens at once on one slide. Also, since more than one horizontal section frequently can be mounted on one slide, the surgeon may rapidly examine successively deeper sections into the tissue block. This technique reduces the number of glass slides utilized and increase the rapidity with which frozen sections can be prepared and examined.

\section{References}

1. Randle HN, Zitelli J, Brodland DG, Roenigk, RK. Histologic preparation for Mohs micrographic surgery - the single section method. J Dermatol Surg Oncol. 1993;19(6): 522-524.

2. Kimyai-Asadi A, Goldberg LH, Nemeth A, Friedman PM, Jih MH. Mohs micrographic surgery for elliptical excisions of skin tumors: a surgical and histologic study. Dermatol Surg. 2004;30(10):1310-1317.

3. Gloster HM. Surgical pearl: large single sections in Mohs micrographic surgery. J Am Acad Dermatol. 2003;49(3):506-508.

4. Gloster HM, Taylor AF. Surgical pearl: the use of multiple different tissue specimens on the same glass slide to enhance the efficiency of frozen section preparation in Mohs micrographic surgery. J Am Acad Dermatol. 1998;39(1):107-108. 